\title{
Simulation Training: An Original Pedagogical Tool to Reduce Maternal and Neonatal Mortality in Africa
}

\author{
Ndour Daouda ${ }^{1 *}$, Gueye Doudou² and Gassama Omar ${ }^{3}$ \\ ${ }^{1}$ Neonatal intensive care unit, Dalal Jamm hospital, Dakar, Senegal \\ ${ }^{2}$ Children's hospital, Dalal Jamm, Dakar, Senegal \\ ${ }^{3}$ Gynecology and obstetrics clinic, Aristide Le Dantec Hospital, Senegal
}

*Corresponding author: Ndour Daouda, Neonatal intensive care unit, Dalal Jamm hospital, Dakar, Senegal.

Received Date: January 07, 2021

Published Date: February 05, 2021

\section{Introduction}

In 2019, 2.4 million children worldwide died during their first month of life. There are approximately 7,000 newborn deaths per day, accounting for $47 \%$ of all under-five deaths, up from $40 \%$ in 1990.

Significant progress has been made in child survival since 1990. Globally, the number of neonatal deaths has increased from 5.0 million in 1990 to 2.4 million in 2019. However, the decline in neonatal mortality from 1990 to 2019 has been slower for newborns than for children under five years of age who have passed the neonatal period.

The proportion of neonatal deaths among children under age 5 is still relatively low in sub-Saharan Africa (36\%), which remains the region with the highest under-five mortality rate in the world. In Europe and North America, where under-five mortality rates are among the lowest of the regions targeted in the sustainable development goals, $54 \%$ of all under-five deaths occur in the neonatal period. An exception is South Asia, where the proportion of neonatal deaths is among the highest (62 percent) despite a relatively high under-five mortality rate [1].

Lack of skilled care is a critical reason why women and newborns continue to die in unacceptably high numbers.

Medical simulation improves knowledge and develops technical and behavioral skills in a safe environment that reproduces real-life situations and environments using dedicated equipment.

\section{Targets}

In order to achieve the objectives of reducing maternal and neonatal mortality in Africa, this training should target as a priority doctors working in the delivery room, nurses, midwives, childcare workers, health care assistants and community workers.

\section{Objectives}

Development of skills to fight against maternal-fetal mortality and morbidity in Africa.

\section{Targeted Competencies}

- Recognize asphyxia at birth and start resuscitation;

- Recognize the need to ventilate and properly conduct mask ventilation;

- $\quad$ Recognize the need for chest compressions and perform them appropriately;

- Have the attitude adapted to one's abilities and know how to call for help;

- $\quad$ Recognize and properly manage postpartum hemorrhage;

- $\quad$ Recognize and properly manage preeclampsia.

\section{Program and Method}

Simulation is a pedagogical method that allows learners to be active and thus optimize the acquisition, integration and subsequent 
reuse of their knowledge. It offers them the possibility of practical learning. Scenarios close to reality put them face to face with clinical situations they are likely to encounter in their professional practice. Thanks to a dynamic simulator dummy, participants are guided in their actions and behaviors by the trainers.

This training includes theoretical reminders, workshops of technical gestures and scenarios reproducing frequent clinical situations requiring the organization and implementation of the first emergency gestures in neonatology and obstetrics.

\section{Pedagogical and Technical Means}

The training will be done by a mobile unit composed of two midwives trained in medical simulation who will travel regularly to each Health Center and each Health Station to provide practical training on essential life-saving procedures for mothers and children before, during and after childbirth.

This training will be repeated and improved (every semester) and has the advantage of being decentralized (agents will be trained in their workplaces), which allows it to be adapted to the context.

The participants will be guided in their actions and behaviors by the trainers.

\section{Evaluation}

A pre- and post-training evaluation will always be offered.

The evaluation will also be done through a satisfaction questionnaire to be completed by the participants at the end of the training. It will focus in particular on the achievement of the pedagogical objectives.

The questionnaires will then be analyzed and the impact of the training on the improvement of the care of mothers and newborns will be evaluated.

\section{Clinical Impact of Simulation}

Over the last ten years, several studies have shown that simulation improves technical and non-technical skills in obstetrical and neonatal emergency situations. In addition, it reinforces the confidence of the health care team.
In addition to Draycott's study of the reduction in neonatal injuries after training shoulder dystocia maneuvers, [2] a larger study of the same group demonstrated a reduction in neonatal complications after the introduction of annual systematic obstetric emergency training incorporating simulation with other traditional teaching methods. Following the introduction of this training, the number of Apgar m 6 to 5 minutes and neonatal hypoxic and ischemic encephalopathies decreased significantly [3].

A program in Tanzania demonstrated the potential of simulation to reduce the occurrence of obstetric complications in countries with few material resources, including improved management of the third stage of labor [4].

Simulation programs have shown significant changes in caregivers and patients. For example, the introduction of a multidisciplinary program on obstetric emergencies was associated with a $45 \%$ decrease in midwife absenteeism, probably due to a perception of better management of difficult situations [5].

\section{Acknowledgement}

None.

\section{Conflict of Interest}

No conflict of interest.

\section{References}

1. WHO (2020) Newborns: improving their survival and well-being.

2. Timothy J Draycott, Joanna F Crofts, Jonathan P Ash, Louise V Wilson, Elaine Yard, et al. (2008) Improving neonatal outcome through practical shoulder dystocia training. Obstet Gynecol 112(1): 14-20.

3. Tim Draycott, Thabani Sibanda, Louise Owen, Valentine Akande, Cathy Winter, et al. (2006) Does training in obstetric emergencies improve neonatal outcome?. BJOG 113(2): 177-182

4. Bjarke Lund Sorensen, Vibeke Rasch, Siriel Massawe, Juma Nyakina, Peter Elsass, et al. (2011) Advanced life support in obstetrics (also) and post-partum hemorrhage : A prospective intervention study in Tanzania. Acta Obstet Gynecol Scand 90(6): 609-614.

5. Jette Led Sørensen, Ellen Løkkegaard, Marianne Johansen, Charlotte Ringsted, Svend Kreiner, et al. (2009) The implementation and evaluation of a mandatory multi-professional obstetric skills training program. Acta Obstet Gynecol Scand 88(10): 1107-1117. 